\title{
Increased circulating microparticles and inflammatory factors aggravate coronavirus disease 2019 (COVID-19)
}

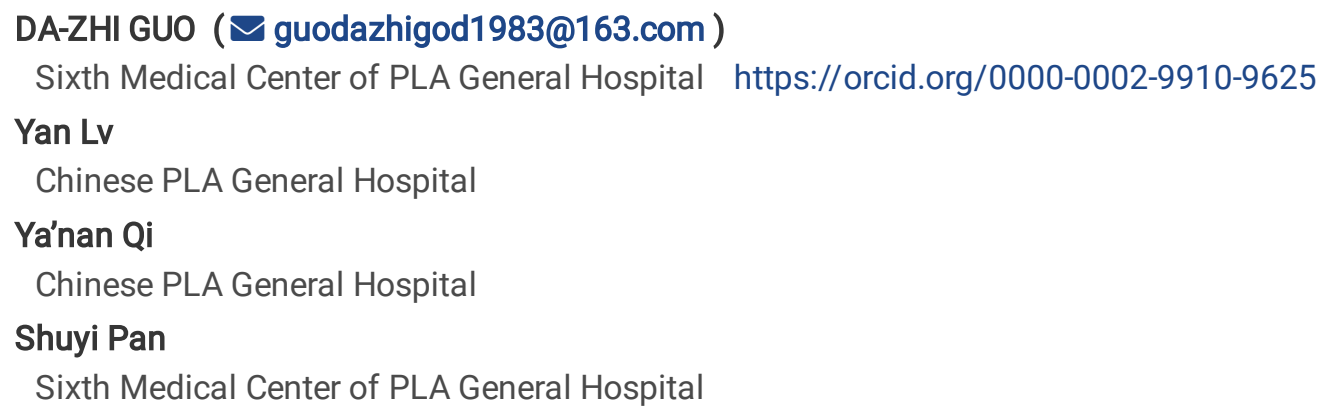

\section{Research article}

Keywords: circulating microparticle, inflammation, interleukin-6, interleukin-8, interleukin-10, COVID-19

Posted Date: April 1st, 2020

DOI: https://doi.org/10.21203/rs.3.rs-19182/v1

License: (c) (i) This work is licensed under a Creative Commons Attribution 4.0 International License. Read Full License 


\section{Abstract}

Background The outbreak of coronavirus disease 2019 (COVID-19) caused by severe acute respiratory syndrome coronavirus 2 (SARS-CoV-2) has posed tremendous challenges to the world's public health. The conditions of some patients can quickly progress to acute respiratory distress syndrome and even death. Current studies suggest that an outbreak of inflammatory response known as cytokine storm may play a key role in the process. This study was performed to investigate the relativity of the production of circulating microparticles (cMPs) and the expression of inflammatory factors (interleukin [IL]-6, IL-8 and IL-10) expression in the blood on the degree of COVID-19.

Methods A total of 89 patients infected with SARS-CoV-2 were divided into four groups, namely, mild, moderate, severe and critically ill groups, according to China's COVID-19 Diagnosis and Treatment Program (6th trial version), and 30 healthy persons were selected as the control group. The groups were compared in terms of cMP production and IL-6, IL-8 and IL-10 levels in their blood, and the correlation between cMP production and the expression of inflammatory factors with the degree of COVID-19 were analysed.

Results Differences in cMP production and IL-6, IL-8 and IL-10 expression levels among the five groups were statistically significant $(P<0.05)$. Pearson correlation analysis showed a positive correlation between CMP production or the expression of IL-6, IL-8 and IL-10 and the degree of COVID-19 (cMP, r=0.981; IL-6, r=0.954; IL-8, r=0.863; IL-10, r=0.895; P<0.05).

Conclusion Increased levels of cMP, IL-6, IL-8 and IL-10 aggravate COVID-19. cMP production and the expression of IL-6, IL-8 and IL-10 may predict the degree of COVID-19 to a certain extent. The early inhibition of cMP overproduction and IL-6, IL-8 and IL-10 expression may be an important therapeutic target for the prevention of COVID-19 progression.

\section{Introduction}

Numerous cases of coronavirus disease 2019 (COVID-19) have occurred in Wuhan City, Hubei Province, China since December 2019. The other parts of China and the world have reported an increasing number of cases with the spread of severe acute respiratory syndrome coronavirus 2 (SARS-CoV-2) [1]. A current research showed that SARS-CoV-2 belongs to the $\beta$-coronavirus genus. Its genetic characteristics are remarkably different from SARS-CoV and Middle East respiratory syndrome (MERS) coronavirus, but its homology with SARS-like coronavirus (bat-SL-CoVZC45) is more than 85\% [2]. Respiratory droplets and close contact are the main routes of transmission of SARS-CoV-2. The incubation period of COVID-19 is 1-14 days but mostly 3-7 days. Respiratory symptoms, such as fever, dry cough and fatigue, are the main clinical manifestations. Severe patients usually have dyspnoea and/or hypoxemia one week after the onset. The conditions of some patients can quickly progress to acute respiratory distress syndrome (SARS), septic shock, difficult-to-correct metabolic acidosis, coagulation dysfunction and multiple organ failure [3]. At present, the mechanism of the sudden deterioration from COVID-19 is unclear, and predictive indicators and effective therapy to prevent its occurrence are not yet available.

The absolute number of lymphocytes in most patients with COVID-19 has decreased, suggesting that this disease mainly affects lymphocytes, especially T lymphocytes [4]. Wang Fusheng's team analysed the peripheral blood cells of patients with COVID-19 and found that the number of CD4+ and CD8+ T cells was reduced, but their activity was excessively enhanced, as shown by the increase of Th17 cells with pro-inflammatory effects in the CD4+ T cells and cytotoxic particles (granulin, granulysin) in the CD8+ T cells [5]. In addition, the levels of interleukin (IL)-2, IL-7, IL-10, granulocyte colony stimulating factor, IFN-y inducible protein 10, monocyte chemokine protein 1, macrophage inflammatory protein 1 alpha and tumour necrosis factor alpha (TNF-a) were substantially increased in the plasma of patients with severe COVID-19 [6]. These studies suggest that an outbreak of inflammatory response known as cytokine storm plays a key role in deterioration caused by COVID-19. Cytokine storm is one of the causes of death in patients with severe and critical pneumonia.

Circulating microparticles (cMPs) are small vesicles with a diameter of less than $1 \mu \mathrm{m}$ and are shed from the cell membrane to blood circulation after cell activation, injury or apoptosis. CMP is involved in several pathophysiological processes, such as procoagulant activity, pro-inflammatory process, endothelial damage, vasoconstriction, vascular growth and immune regulation. cMP production is remarkably increased in diseases, such as diabetes, cardiovascular disease, AIDS, infection and chronic inflammation $[7,8]$. Furthermore, cMP production stimulates the increase in the levels of inflammatory factors, such as IL-1, IL-6, 
IL-8 and TNF- $a$. These cytokines in turn further activates inflammation, which increases the level of cMPs and therefore forms the 'inflammatory waterfall' response [9]. However, whether cMP production is involved in the pathophysiology of COVID-19 remains unclear.

In this study, we grouped patients with COVID-19 from mild to critically ill and detected their cMP production and expression of inflammatory factors (IL-6, IL-8 and IL-10) in the blood. Moreover, we analysed the correlation of cMPs and inflammatory factors with the severity of COVID-19 to obtain effective indicators and therapeutic targets and predict and prevent the occurrence of this disease.

\section{Methods}

\section{Patient population}

A total of 119 patients with COVID-19 were enrolled from January 2020 to February 2020. The diagnostic criteria based on China's COVID-19 Diagnosis and Treatment Program (trial version 6) are as follows [10]: (1) Real-time fluorescent reverse-transcription polymerase chain reaction detection of SARS-CoV-2 nucleic acid is positive, and (2) viral gene sequencing is highly homologous with SARS-CoV-2. The following are the clinical classification diagnostic criteria of COVID-19 [10]. (1) Mild: The clinical symptoms are slight, and no manifestations of pneumonia are seen in the lung computed tomography (CT). (2) Moderate: Fever, respiratory symptoms and manifestations of pneumonia are seen in the lung CT. (3) Severe: The patient meets one of the following criteria: shortness of breath, $\mathrm{RR} \geq 30$ beats/min; $\mathrm{SpO} 2 \leq 93 \%$ at rest state; $\mathrm{PaO} 2 / \mathrm{FiO} 2 \leq 300 \mathrm{mmHg}(1 \mathrm{mmHg}=0.133 \mathrm{kPa}) ; \mathrm{PaO} 2 / \mathrm{FiO} 2$ at high altitudes (above $1000 \mathrm{~m}$ ) should be corrected according to the formula: PaO2/FiO2x (atmospheric pressure $\mathrm{mmHg} / 760$ ); or lung CT showed that lesions progressed by more than $50 \%$ within $24-48 \mathrm{~h}$. (4) Critically ill: The patient meets one of the following criteria: respiratory failure occurs, and mechanical ventilation is required; Shock; organ failure and need to enter ICU treatment. The patients with COVID-19 were divided into mild (group $A, n=25$ ), moderate (group $B, n=26$ ), severe (group $C, n=20$ ) and critically ill (group $D, n=18$ ) groups. Thirty healthy persons were enrolled as the control group. This study was approved by the Medical Ethics Committee of the Sixth Medical Centre of the General Hospital of the Chinese People's Liberation Army. Patients and their families were informed and signed informed consent.

\section{Data collection}

We extracted the clinical data of all enrolled subjects from the medical records. The data included age, gender, body mass index (BMI) and history of basic diseases (hypertension, diabetes, hyperlipidemia and coronary heart disease). Venous blood samples were obtained within $24 \mathrm{~h}$ after admission for routine laboratory tests, separation and detection of microparticles (MPs) in plasma and detection of IL-6, IL-8 and IL-10 expression on serum. Lung CT examinations were simultaneously performed. All body fluid samples were collected and tested in accordance with the requirements of China's COVID-19 Patients' Blood Test Sample Preservation and Management Scheme (Trial). All operations were performed by professionals.

\section{Plasma microparticle separation}

We followed the procedure previously described [12]. All reagents and solutions used for MP analysis were sterile and filtered ( 0.2 $\mu \mathrm{m}$ filter). Heparinised blood was centrifuged for $5 \mathrm{~min}$ at $1500 \times \mathrm{g}$. The supernatant made of $0.2 \mathrm{M}$ EDTA was prepared by diluting the supernatant in a ratio of 2:5 with $0.5 \mathrm{M}$ solution of tetrasodium EDTA prepared in PBS to diminish ex vivo MP aggregation. The supernatant was then centrifuged at $15,000 \times g$ for $30 \mathrm{~min}$ for the pelleting of the few remaining platelets and cell debris. Supernatant $(250 \mathrm{ml})$ was parcelled among centrifuge tubes, added with $4 \mathrm{ml}$ of PBS and centrifuged at 100,000 $\times g$ for 60 min (3-4 tubes per experiment were used). MPs were found in a loose pellet at the bottom of the centrifuge tubes.

\section{Flow cytometry analysis of blood-borne MPs}

Flow cytometry was performed with a 10-color FACSCanto (Becton Dickinson, San Jose, CA) and standard acquisition software. Counting was based on light scattering, and the gate was set to include particles ranging from $0.3 \mu \mathrm{m}$ to $6.5 \mu \mathrm{m}$ excluding debris normally present in the buffer. MPs were stained with annexin $V$ antibody and analysed. In addition to the sample, microbeads with $0.3 \mu \mathrm{m}$ diameter from Sigma and microbeads with 1.0x, 3.0x and $6.5 \mu \mathrm{m}$ diameter from Spherotech (Lake Forest, IL) were used for the initial setup for the estimation of the diameters of the MPs before each experiment. The analysis involved

Page $3 / 9$ 
fluorescence subtraction and concentration. True negative controls were established under the same conditions by using isotypematched irrelevant antibodies. Forward and side scatter were set to log gain. MPs were calculated by calculating the ratio of beads and analysing the exact volume of MP in plasma.

\section{Detection of serum IL-6, IL-8 and IL-10}

Whole blood sample in the serum separation tube was left at room temperature for $2 \mathrm{~h}$ and then centrifuged at $1000 \times g$ for 20 min. The supernatant was obtained. Enzyme-linked immunosorbent assay was performed in strict accordance with the manufacturer's instruction (R \& D Systems).

\section{Statistical analysis}

We used SPSS 19.0 statistical software package for statistical processing. Continuous data were shown as mean \pm standard deviation $(\overline{\mathrm{x}} \pm \mathrm{s})$. Enumeration data were shown as frequency and percentage. Comparisons between groups were performed using $\mathrm{x}^{2}$ test or Fisher exact probability method. Pairwise comparison was performed by $\mathrm{Q}$ test. Correlation analysis was performed by Pearson method. A P value of $<0.05$ was considered significant.

\section{Results}

\section{Baseline characteristics of patients}

The five groups had no significant difference in gender; BMI; history of hypertension, diabetes and hyperlipidemia; and levels of creatinine ( $\mathrm{Cr}$ ), blood urea nitrogen (BUN), alanine aminotransferase (ALT), creatinine kinase and D-dimer $(P>0.05)$. However, significant differences were observed in age and history of coronary heart disease $(P<0.05)$. Patients in the severe and critically ill groups were older and had a higher proportion of patients with coronary heart disease (Table 1).

\section{Comparison of MPs production in plasma}

The mean MPs in the plasma of a healthy person was $1851.53 \pm 30.13 / \mu l$. The number of MPs in patients with COVID-19 was significantly higher than that in the control group $(P<0.05)$. Moreover, a significant difference was observed in the number of MPs in patients with different levels of severity of COVID-19 $(P<0.05)$. More MPs were produced in patients with a severe COVID-19 (Table 2).

\section{Comparison of IL-6, IL-8 and IL-10 expression in serum}

The expression of IL-6, IL-8 and IL-10 in the serum of patients with COVID-19 increased significantly compared with the control group $(\mathrm{P}<0.05)$. Moreover, the expression of IL-6, IL-8 and IL-10 in patients with different levels of severity of COVID-19 was also significantly different $(P<0.05)$. Higher levels of IL-6, IL-8 and IL-10 were expressed in patients with a more severe COVID-19 (Table $3)$.

\section{Correlation of MP production and expression of IL-6, IL-8 and IL-10 with the severity of COVID-19}

Pearson correlation analysis results showed a remarkably positive correlation of MP production and the expression of IL-6, IL-8 and IL-10 with the severity of COVID-19 (Table 4).

\section{Discussion}

The main clinical feature of severe COVID-19 is the appearance of acute respiratory distress syndrome [6]. Wang et al. [5] performed biopsy of lung tissue from patients with COVID-19 and found that the pathological features of COVID-19 are extremely similar to those of SARS and MERS, which are characterised by the desquamation of lung cells and the formation of transparent membranes. Liu Liang et al. [12] performed an autopsy of a patient with COVID-19 and found that the lungs of the deceased showed obvious inflammatory damage and exudative changes, a large number of sticky secretions overflowed from the alveolar section and fibrous cords were formed in the lung tissue. These studies suggested that inflammatory response is closely related to the progression of COVID-19. The purpose of this study was to investigate the correlation between the factors that mediate 
inflammatory response, such as cMPs and cytokines (IL-6, IL-8 and IL-10) and the severity of COVID-19 and determine whether the amount of cMPs and the expression of cytokines (IL-6, IL-8 and IL-10) can be used as indicators of the severity of COVID-19.

Inflammation is a protective immune response that helps clear the source of infection. However, excessive inflammation can cause immune damage as well. cMPs are vesicles produced by the remodelling and budding of cell membranes and are mainly composed of phospholipids and proteins. However, their internal components are not completely clear. cMPs are divided into platelet microparticles (PMPs), granulocyte MPs, erythrocyte microparticles (endothelial cell microparticles, EMPs) and lymphocyte-derived MPs according to their source [13]. Zafrani et al. found that cMP production was remarkably reduced in calpain knock-out transgenic mice, and the risk of inflammation, diffuse intravascular coagulation (DIC) and organ damage was greatly reduced. However, they found that the latter caused coagulation disorders and increased mortality when they injected cMPs from wild type mice with septicaemia into calpain knock-out transgenic mice [14], indicating that MPs are directly involved in calpain-mediated inflammatory injury during the course of sepsis. PMPs can stimulate the production of inflammatory factors, such as IL-1, IL-6, IL-8 and TNF-a. These cytokines, in turn, further activate inflammation and allowing cells to produce more cMPs, which exacerbate inflammatory response [15]. EMPs increase CD105 expression and decrease CD31 expression in the early stage of DIC and are directly related to the occurrence of DIC [16]. NMPs, especially those carrying high concentrations of IL-1 $\beta$, are an important cause of tissue damage in several diseases [17]. This previous research shows that cMPs are closely related to the occurrence and development of inflammation and infection. In the present study, we isolated and detected cMPs in the blood of patients with COVID-19 of different levels of severity and of healthy persons. We found that CMPs in the blood of patients with COVID-19 were remarkably increased compared with those in healthy persons, and the number of MPs increased with the severity of COVID-19; thus, a remarkably positive correlation exists between them. The increase in cMP production is involved in the progression of COVID-19, and the number of cMPs may reflect the severity of COVID-19.

Recent studies have shown that MPs contain cellular receptors, cytoplasmic proteins, nucleic acids (RNA, microRNA and DNA) and cytokines. Among them, cytokines may be the key factor for mediating lung inflammation storms [18]. Alveolar macrophages in patients with severe pneumonia are stimulated to release a large amount of IL-6 and TNF-a in the early stages of infection [19]. The elevation of IL-6 levels can limit inflammation and protect the body to a certain extent at the early stage of disease. However, the excessive release of IL- 6 can induce fibrinogen activation, activate coagulation factors, promote microthrombosis in inflammation sites and inhibit endothelial repair, which can make blood vessels highly permeable and further aggravate inflammatory lung injury [20]. IL-8 is the strongest neutrophil chemotactic and activating factor and has dual immune effects on the body. Low levels of IL-8 can protect against infection, and excessive IL-8 can induce inflammatory response. Higher IL-8 levels indicate a poor prognosis [21]. IL-10 is an anti-inflammatory factor that can down-regulate the host's immune response through T helper cells and inhibit the overexpression of pro-inflammatory cytokines. However, excessive anti-inflammatory response can inhibit the body's immune function, which is not conducive to the elimination of exogenous bacteria and viruses and results in a poor prognosis for patients [22]. We found that the expression of serum IL-6, IL-8 and IL-10 in patients with COVID-19 were higher than those in the control group and increased with the worsening of the disease. This result indicated that IL-6, IL-8 and IL-10 are involved in the inflammatory response process of COVID-19 and have important clinical significance for predicting the prognosis of patients. We speculated that IL- 6 and IL-8 may play a role in promoting inflammation damage and IL-10 may play a role in suppressing the body's immune defence. In addition, we also found that older patients and patients with history of coronary heart disease accounted for a higher proportion in the severe and critically ill groups, further suggesting that the elderly and those with cardiac insufficiency and decreased immune function are more susceptible to the 'inflammation attack'.

Alveoli are the main site of gas exchange in the lungs and the functional units of the lungs and are composed of types I and II alveolar cells, as well as alveolar macrophages and surfactants. We speculated that a large number of SARS-CoV-2 may induce cMP production to increase through multiple mechanisms (hypoxia, oxidative stress, cell activation, calcium influx, apoptosis, etc.) when these viruses invade type I and type II alveolar cells. Hence, alveolar cells and/or immune cells are activated to secrete TNF, IL-6, IL-8, IL-10 and other cytokines. Thus, the body's immune defence and anti-inflammatory balance are broken, and thus damage caused by pneumonia worsens. These cytokines further recruit inflammatory cells to the infection site and destroy type II alveolar cells, eventually leading to respiratory failure. We believe that increased cMP production may be the cause or result of the exacerbation of COVID-19. 


\section{Conclusion}

Our study showed that cMP production and the expression of cytokine factors (IL-6, IL-8 and IL-10) increased with the severity of COVID-19 and are positively correlated with the severity of COVID-19. cMP production and the expression of IL-6, IL-8 and IL-10 can be used as markers for evaluating the severity of COVID-19. The early inhibition of cMP overproduction and the expression of IL-6, IL-8 and IL-10 may be important therapeutic targets for preventing the progression of COVID-19. However, some limitations exist in our study. (1) The requirements for specimen collection and testing are very strict because COVID-19 is highly infectious. The number of patients enrolled in this study is small, and selective bias and measurement bias may occur. (2) Our results showed that patients in severe and critically ill groups were older and had increased white blood cells. However, the poor prognosis of these groups may be related to the poor immune function in the elderly population and could be acquired secondary to bacterial pneumonia. (3) This study did not further study the relationship between the outcome of each group and the dynamic changes in cMP production and the expression of IL-6, IL-8 and IL-10. (4) This study was not able to explain whether a correlation exists between the increase in cMP production and the sources of increased expression levels of IL-6, IL-8 and IL-10. In the future, we need to further study the role and mechanism of MPs and inflammation in the pathophysiology of COVID-19.

\section{Declarations}

\section{Acknowledgements}

Sincere gratitude is extended to the doctors, nurses and participants in Wuhan for fighting the new coronavirus infection and for their efforts and cooperation.

\section{Authors' contributions}

Study design and funding: Dazhi Guo, data collection: Dazhi Guo and Yanan Qi, data analysis and interpretation: Dazhi Guo, statistics: Yan Lv and Shuyi Pan, drafting article: Dazhi Guo, approval of article: all authors.

\section{Availability of data and materials}

The datasets used during the present study are available upon reasonable request to the corresponding author.

\section{Ethics approval and consent to participate}

This study was approved by the Medical Ethics Committee of the Sixth Medical Centre of the General Hospital of the Chinese People's Liberation Army (No. 20200313). All patients and their families were informed and signed informed consent.

\section{Patient consent for publication}

Data of the enrolled subjects were de-identified. This study was approved by the institutional review board.

\section{Competing interests}

The authors declare no conflicts of interest.

\section{References}


1. Lai CC, Shih TP, Ko WC, Tang HJ, Hsueh PR. Severe acute respiratory syndrome coronavirus 2 (SARS-CoV-2) and coronavirus disease-2019 (COVID-19): The epidemic and the challenges. Int J Antimicrob Agents 2020, 17:105924. doi:

10.1016/j.ijantimicag.2020.105924.

2. Fan Y, Zhao K, Shi ZL, Zhou P. Bat Coronaviruses in China. Viruses 2019, 11(3). pii: E210.

3. Cheng ZJ, Shan J. 2019 Novel coronavirus: where we are and what we know. Infection 2020, 18. doi: 10.1007/s15010-02001401-y.

4. Wang D, Hu B, Hu C, Zhu F, Liu X, Zhang J, Wang B, Xiang H, Cheng Z, Xiong Y, Zhao Y, Li Y, Wang X, Peng Z. Clinical Characteristics of 138 Hospitalized Patients With 2019 Novel Coronavirus-Infected Pneumonia in Wuhan, China. JAMA 2020, 7. doi: $10.1001 /$ jama.2020.

5. Xu Z, Shi L, Wang Y, et al. Pathological findings of COVID-19 associated with acute respiratory distress syndrome. Lancet Respir Med 2020, 18. pii: S2213-2600(20)30076-X. doi: 10.1016/S2213-2600(20)30076-X.

6. Huang C, Wang Y, Li X, et al. Clinical features of patients infected with 2019 novel coronavirus in Wuhan, China. Lancet 2020, 395(10223): 497-506.

7. Provost P. The clinical significance of platelet microparticle-associated microRNAs. Clin Chem Lab Med 2017, 55(5): $657-666$.

8. Alexandru N, Badila E, Weiss E, Cochior D, Stępień E, Georgescu A. Vascular complications in diabetes: Microparticles and microparticle associated microRNAs as active players. Biochem Biophys Res Commun 2016, 472(1): 1-10.

9. Thom SR, Bhopale VM, Yu K, Huang W, Kane MA, Margolis DJ. Neutrophil microparticle production and inflammasome activation by hyperglycemia due to cytoskeletal instability. J Biol Chem 2017, 292(44): 18312-18324.

10. National Health Commission of the People's Republic of CHina. China's COVID-19 Diagnosis and Treatment Program (trial version 6). 2020. Beijing, China.

11. Thom SR, Yang M, Bhopale VM, Huang S, Milovanova TN. Microparticles initiate decompression-induced neutrophil activation and subsequent vascular injuries. J Appl Physiol (1985) 2011, 110(2): 340-51.

12. Liu Qian, Wang Rongshuai, Qu Guoqiang, et al. Gross observation of anatomy of a dead coronal system of a new coronavirus pneumonia. Journal of Forensic Medicine 2020, 36 (1): 1-3.

13. Nomura S $₫$ Ozaki Y邓lkeda Y.Function and role of microp- articles in various clinical setings. Thromb Res 2008, 123(1): 8-23

14. Zafrani L, Gerotziafas G, Byrnes C, Hu X, Perez J, Lévi C, Placier S, Letavernier E, Leelahavanichkul A, Haymann JP, Elalamy I, Miller JL, Star RA, Yuen PS, Baud L. Calpastatin controls polymicrobial sepsis by limiting procoagulant microparticle release. Am J Respir Crit Care Med 2012, 185(7): 744-755.

15. Aksu K, Donmez A, Keser G. Inflammation-induced thrombosis: mechanisms, disease associations and management. Curr Pharm Des 2012, 18(11): 1478-1493.

16. Zafrani L, Ince C, Yuen PS. Microparticles during sepsis: target, canary or cure. Intensive Care Med 2013, 39(10): $1854-1856$.

17. Thom, S.R., Bhopale, V.M., Yu, K. \& Yang, M. Provocative decompression causes diffuse vascular injury in mice mediated by microparticles containing interleukin-1 $\beta$. J Appl Physiol 2018, 125, 1339-1348.

18. Pap E『Pálinger E『Pásztói M『et al.Highlights of a new type of intercelular communication:microvesicle-based information transfer. Inflamm Res 2009, 58(1): 1-8.

19. Chen F®Zhang Y®Zhang C. Effect of ambroxol on the concentration of cefotaxime in the bronchoalveolar lavage fluid of rats with pulmonary fibrosis. Exp Ther Med 2015, 9(2): 539-542.

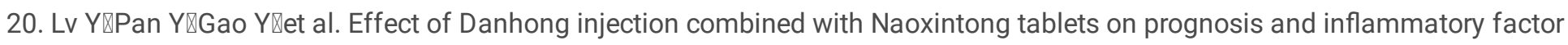
expresion in acute coronary syndrome patients under- going percutaneous coronary intervention. Acta Cardiol Sin 2015, 31(4): 301-307.

21. Butt Y, Kurdowska A, Allen TC. Acute Lung Injury: A Clinical and Molecular Review. Arch Pathol Lab Med 2016, 140(4): $345-50$.

22. Tyrrell C, Mckechnie SR, Beers M F, et al. Differential alveolar epithelial injury and protein expresion in pneumococal pneumonia. Exp Lung Res 2012, 38(5): 266-276.

\section{Tables}


Table 1 Baseline characteristics of patients in the five groups

\begin{tabular}{|c|c|c|c|c|c|c|c|}
\hline $\begin{array}{l}\text { Basic } \\
\text { information }\end{array}$ & Control & Group A & Group B & Group C & Group D & $\mathrm{F} / \mathrm{x} 2$ & $\begin{array}{l}\mathrm{P}- \\
\text { value }\end{array}$ \\
\hline Number (n) & 30 & 25 & 26 & 20 & 18 & - & \\
\hline $\begin{array}{l}\text { Female/male } \\
\text { (n) }\end{array}$ & $14 / 16$ & $12 / 13$ & $13 / 13$ & $9 / 11$ & $8 / 10$ & 0.184 & 0.996 \\
\hline Age (years) & $49.73 \pm 15.76$ & $46.76 \pm 15.62$ & $47.19 \pm 13.53$ & $57.65 \pm 15.60$ & $60.56 \pm 14.09$ & 3.747 & 0.007 \\
\hline BMI $\left(\mathrm{kg} / \mathrm{mm}^{2}\right)$ & $21.77 \pm 1.37$ & $21.53 \pm 2.20$ & $21.01 \pm 1.41$ & $21.80 \pm 1.40$ & $21.94 \pm 1.39$ & 1.268 & 0.287 \\
\hline $\begin{array}{l}\text { Hypertension } \\
\text { (n) }\end{array}$ & 10 & 8 & 8 & 9 & 8 & 1.831 & 0.767 \\
\hline Diabetes (n) & 8 & 6 & 7 & 10 & 10 & 8.358 & 0.079 \\
\hline $\begin{array}{l}\text { Coronary } \\
\text { heart disease } \\
\text { (n) }\end{array}$ & 3 & 2 & 3 & 5 & 4 & 11.727 & 0.02 \\
\hline $\begin{array}{l}\text { Hyperlipidemia } \\
\text { (n) }\end{array}$ & 10 & 8 & 8 & 6 & 5 & 0.185 & 0.996 \\
\hline $\mathrm{Cr}(\mathrm{mmol} / \mathrm{L})$ & $72.23 \pm 5.76$ & $71.88 \pm 26.11$ & $73.12 \pm 5.81$ & $72.65 \pm 6.41$ & $72.39 \pm 6.26$ & 0.143 & 0.966 \\
\hline BUN (mmol/L) & $6.27 \pm 0.65$ & $6.30 \pm 0.66$ & $6.29 \pm 0.78$ & $6.35 \pm 0.83$ & $6.20 \pm 0.66$ & 0.105 & 0.981 \\
\hline $\begin{array}{l}\text { WBC count } \\
\left(\times 10^{9} / \mathrm{L}\right)\end{array}$ & $6.19 \pm 1.30$ & $5.94 \pm 1.87$ & $4.77 \pm 1.91$ & $7.18 \pm 3.02$ & $11.98 \pm 3.37$ & 30.109 & 0.000 \\
\hline $\mathrm{ALT}(\mathrm{U} / \mathrm{L})$ & $26.05 \pm 10.93$ & $26.32 \pm 10.05$ & $28.19 \pm 11.44$ & $26.69 \pm 7.77$ & $26.74 \pm 9.46$ & 0.177 & 0.950 \\
\hline $\begin{array}{l}\text { Creatinine } \\
\text { kinase (IU/L) }\end{array}$ & $89.99 \pm 6.57$ & $90.08 \pm 13.56$ & $91.3 \pm 7.57$ & $93.98 \pm 11.88$ & $93.63 \pm 6.93$ & 0.874 & 0.482 \\
\hline D-dimer & $384.44 \pm 17.71$ & $384.20 \pm 19.18$ & $381.45 \pm 16.73$ & $404.32 \pm 19.56$ & $420.38 \pm 144.53$ & 1.739 & 0.146 \\
\hline
\end{tabular}

Table 2 Comparison of MP production in the plasma of patients in the five groups

\begin{tabular}{ccc}
\hline Group & Cases $(\mathrm{n})$ & MP production \\
\hline Group A & 25 & $2044.64 \pm 31.20^{\mathrm{bcdt}}$ \\
Group B & 26 & $2257.12 \pm 27.50^{\mathrm{acdt}}$ \\
Group C & 20 & $2557.65 \pm 31.00^{\mathrm{abdt}}$ \\
Group D & 18 & $2933.44 \pm 104.07^{\mathrm{abct}}$ \\
Control & 30 & $1851.53 \pm 30.13$ \\
\hline $\mathrm{X}^{2}$ & & 1693.136 \\
P value & & 0.000 \\
\hline
\end{tabular}

Note: Compared with control group, ${ }^{\mathrm{t}} \mathrm{P}<0.05$; compared with group $\mathrm{A},{ }^{\mathrm{a}} \mathrm{P}<0.05$; compared with group $\mathrm{B}$, ${ }^{b} \mathrm{P}<0.05$; compared with group $\mathrm{C},{ }^{\mathrm{c}} \mathrm{P}<0.05$; compared with group $\mathrm{D},{ }^{\mathrm{d}} \mathrm{P}<0.05$.

Table 3 Comparison of IL-6, IL-8 and IL-10 expression in the serum of patients in the five groups

\begin{tabular}{lllll}
\hline Group & Cases $(\mathrm{n})$ & IL-6 $(\mathrm{pg} / \mathrm{ml})$ & $\mathrm{IL}-8(\mathrm{pg} / \mathrm{ml})$ & $\mathrm{IL}-10(\mathrm{pg} / \mathrm{ml})$ \\
\hline Group A & 25 & $9.60 \pm 1.64^{\mathrm{bcdt}}$ & $258.39 \pm 35.46^{\mathrm{bcdt}}$ & $5.10 \pm 0.76^{\mathrm{bcdt}}$ \\
Group B & 26 & $12.92 \pm 1.89^{\mathrm{acdt}}$ & $302.49 \pm 48.066^{\mathrm{acdt}}$ & $6.02 \pm 0.65^{\mathrm{acdt}}$ \\
Group C & 20 & $34.57 \pm 13.69^{\mathrm{abdt}}$ & $413.65 \pm 92.21^{\mathrm{abdt}}$ & $9.23 \pm 1.06^{\mathrm{abdt}}$ \\
Group D & 18 & $103.28 \pm 34.68^{\mathrm{abct}}$ & $601.61 \pm 95.72 \mathrm{abct}$ & $11.64 \pm 2.15^{\mathrm{abct}}$ \\
Control & 30 & $3.78 \pm 1.63$ & $209.00 \pm 27.33$ & $4.12 \pm 0.72$ \\
\hline $\mathrm{X}^{2}$ & 581.458 & 137.083 & 175.281 \\
P value & 0.000 & 0.000 & 0.000 \\
\hline
\end{tabular}

Note: Compared with control group, ${ }^{\mathrm{t}} \mathrm{P}<0.05$; compared with group $\mathrm{A},{ }^{\mathrm{a}} \mathrm{P}<0.05$; compared with group $\mathrm{B}$, ${ }^{b} \mathrm{P}<0.05$; compared with group $\mathrm{C},{ }^{\mathrm{c}} \mathrm{P}<0.05$; compared with group $\mathrm{D},{ }^{\mathrm{d}} \mathrm{P}<0.05$.

Table 4 Correlation of MP production and the expression of IL-6, IL-8 and IL-10 with the severity of COVID-19 


\begin{tabular}{ccc}
\hline Inflammatory factors & \multicolumn{3}{c}{ Severity of COVID-19 } \\
\cline { 2 - 3 } & r value & P value \\
\hline MPs production & 0.981 & 0.000 \\
IL-6 & 0.954 & 0.000 \\
IL-8 & 0.863 & 0.000 \\
IL-10 & 0.895 & 0.000 \\
\hline
\end{tabular}

